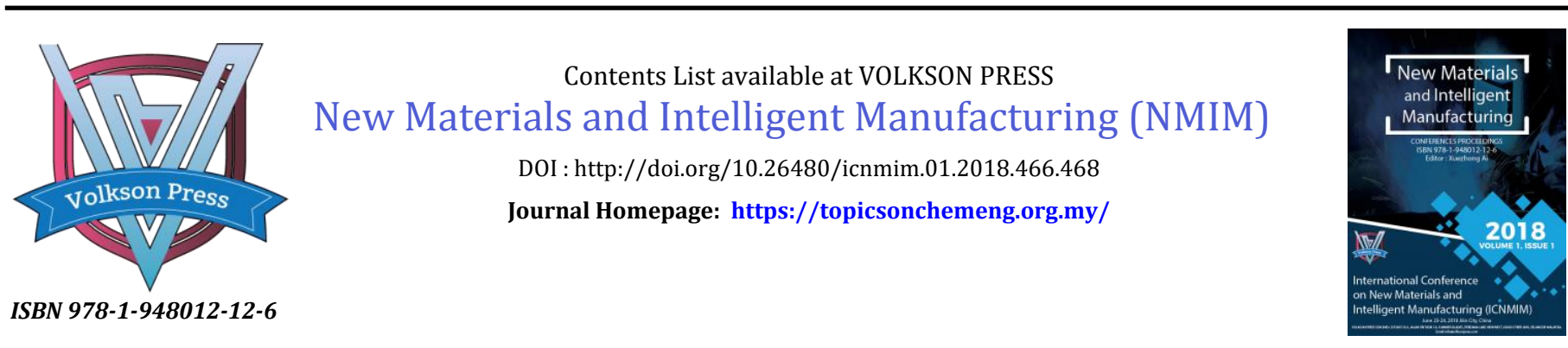

\title{
VARIATION OF PARAMETERS METHOD FOR MICRO-POLAR FLUID THROUGH A CBM POROUS MEDIA
}

\author{
Gao Dapeng ${ }^{1,2, *}$, Guo Zhidong³, Wang Tianjiao4, Liu Yuewu1,2,*, Hou Shaoji1 \\ ${ }^{1}$ Institute of Mechanics, Chinese Academy of Sciences, Beisihuanxi street, Beijing, China \\ ${ }^{2}$ School of Engineering Science, University of Chinese Academy of Sciences, Beisihuanxi street, Beijing, China \\ ${ }^{3}$ PetroChina Coalbed Methane Company Limited, Taiyanggongzhong street, Beijing, China \\ ${ }^{4}$ CNPC Beijing Oilfield Machinery Co., LTD, Nengyuannan street, Beijing, China \\ *Corresponding author email: liuyw@imech.ac.cn; gaodapeng@imech.ac.cn
}

This is an open access article distributed under the Creative Commons Attribution License, which permits unrestricted use, distribution, and reproduction in any medium, provided the original work is properly cited.

\section{ARTICLE DETAILS}

\section{Article History:}

Received 26 June 2018

Accepted 2 July 2018

Available online 1 August 2018

\section{ABSTRACT}

In this article, we adopt the variation of parameters method (VPM) to get the approximate solution of micro-polar fluid through a porous channel which is a rectangular domain with infinite length and bounded by two moving porous walls. To investigate characteristic parameter Reynolds number, the non-dimensional wall expansion rate how to influence of the stream wise velocity and micro-rotation. An approximate analytical solution can be obtained by VPM that the solution may be for some parameter values, which results are effortless to calculate. The proposed method is different from any discretization, transformation and restrictive assumptions, which is supported by numerical results and show excellent agreement. It illustrates VPM is a powerful computational mathematics development tool by which finding the approximate analytical solutions for a system of nonlinear differential equation.

\section{KEYWORDS}

Micro-polar fluid, Approximate analytical methods, VPM, Porous channel.

\section{INTRODUCTION}

In studying the micro-polar fluid through a porous channel with expanding or contracting walls, which has great importance in heavy oil and biological sciences. These types of flows have many critical applications in engineering. Because of which study of these types of streams attracted many researchers from around the world, and they contributed to this work. The solution of flow in porous boundary channel has been done by Berman, who introduced a way to simplify the NavierStocks equation an ordinary differential equation based on the assumption that the fluid injection through the porous boundary [1]. His study has ushered in a new epoch for many researchers.

The flow of the Newtonian fluid through expanding or contracting walls have been well studied and discussed [2-4] numerical method or analytically. Dauenhauer and Majdalani adopted self-similar reduction to simplify the equation and obtained both numerical and asymptotical solutions for the different permeability Reynolds numbers for Newtonian fluid through expanding porous walls [2]. It is made up of White as of form attribute on Dauenhauer and Majdalani to the new analytic Stokes solutions [3]. Asghar put forward a series of definitions and methods to reduce the partial differential equations on account of the combination of the underlying symmetry structure and conservation laws; then the complex system is reduced to an easy to handle second-order ordinary differential equation system, it is independent of limit on any physical parameters [4].

A group researchers discussed the solution for two-dimensional viscous flow in a porous channel with expanding or contracting walls using Liegroup method [5,6]. Nevertheless, the micro-polar flow through the expanding porous walls was not finished by Lie group despite that of a study and micro-polar fluid the combination of micro-rotation velocity and gave the similarity transformation similar to what Srinivasacharya and Ramana Murthy proposed [7,8]. The principle of Lie-group of simplified the Non-Newtonian micro-polar fluid through the expanding or contracting porous channel was discussed in a research paper [9].

Motive by above works, in this article we take advantage of the similar transform in both space and time to reduce the governing equation of the flow and then to solve it with an instrumental technique called a variation of parameters method (VPM). The results showed that the VPM is more accurate and provides such results which are nearer to numerical simulation. In the end, we analyzed the micro-polar flow phenomenon in different Reynolds number, channel expansion rate, and micro-rotation parameter.

\section{MATHEMATICAL MODEL}

In this paper, incompressible, isothermal and unsteady micro-polar fluid flow is considered in a rectangular duct of infinite length porous semiinfinite channel, which contains two permeable walls, from where the fluid can enter or exit during successive expansions/contractions. The width between the porous walls is far less than the length of the channel. One end of the channel is closed by a complicated solid membrane. The walls the suction or injection velocity and expand or contract uniformly at a time-dependent rate, and injection velocity is assumed to be independent of position.

\subsection{Governing Equations}

Assume that $x$ and $y$ are coordinate axes parallel and perpendicular to the porous channel walls, respectively, $u, v$ are the velocity components in the $x$ and $y$ directions and $N$ is micro-rotation, respectively. Under these 
assumptions, the governing equations are expressed as follows:

$\frac{\partial u}{\partial x}+\frac{\partial v}{\partial y}=0$

$\frac{\partial u}{\partial t}+u \frac{\partial u}{\partial x}+v \frac{\partial u}{\partial y}=-\frac{1}{\rho} \frac{\partial P}{\partial x}+\left(v+\frac{\kappa}{\rho}\right)\left(\frac{\partial^{2} u}{\partial x^{2}}+\frac{\partial^{2} u}{\partial x^{2}}\right)+\frac{\kappa}{\rho} \frac{\partial N}{\partial y}$

$\frac{\partial v}{\partial t}+u \frac{\partial v}{\partial x}+v \frac{\partial v}{\partial y}=-\frac{1}{\rho} \frac{\partial P}{\partial y}+\left(v+\frac{\kappa}{\rho}\right)\left(\frac{\partial^{2} v}{\partial x^{2}}+\frac{\partial^{2} v}{\partial x^{2}}\right)-\frac{\kappa}{\rho} \frac{\partial N}{\partial x}$

$\rho j\left(\frac{\partial N}{\partial t}+u \frac{\partial N}{\partial x}+v \frac{\partial N}{\partial y}\right)=-\kappa\left(2 N+\frac{\partial u}{\partial y}-\frac{\partial v}{\partial x}\right)+\gamma\left(\frac{\partial N}{\partial y^{2}}+\frac{\partial^{2} N}{\partial x^{2}}\right)$

The boundary conditions as follows:

$u(x,-a)=0, v(x,-a)=-a^{\prime}(t), N(x,-a)=0$

$u(x, a)=0, N(x, a)=0$

In order to facilitate the analysis, the above governing equations and boundary conditions are represented by the stream function.

$u=\frac{\partial \psi}{\partial y} v=-\frac{\partial \psi}{\partial x}$

And following dimensionless variables are introduced as:

$$
\begin{aligned}
& \bar{u}=\frac{u}{v_{w}}, \bar{v}=\frac{v}{v_{w}}, \bar{x}=\frac{x}{a}, \bar{y}=\frac{y}{a} \\
& \bar{t}=\frac{t}{a / v_{w}}, \bar{\psi}=\frac{\psi}{a v_{w}}, \bar{P}=\frac{P}{\rho v_{w}^{2}}, \bar{N}=\frac{a N}{v_{w}}
\end{aligned}
$$

Substituting (6-7) into (2-4), the governing equations become dimensionless form of the Governing Equations:

$\overline{\psi_{y t}^{-}}+\overline{\psi_{y}^{-}} \bar{\psi}_{y x}^{-}-\overline{\psi_{x}^{-}} \bar{\psi}_{y y}^{-}+\overline{P_{x}^{-}}-\frac{1}{\operatorname{Re}}\left(\alpha \bar{\psi}_{y}^{-}+\partial \bar{\psi}_{y y}^{--}\right)-\frac{1}{\operatorname{Re}}(1+K)\left(\overline{\psi_{y x x}^{-}}+\partial \bar{\psi}_{y y y}^{-}\right)-\frac{K}{\operatorname{Re}} N_{y}=0$

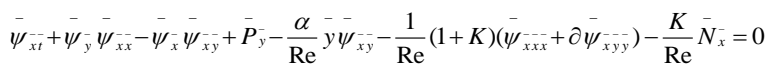

$\left.\bar{N}_{t}^{-}+\bar{\psi}_{y}^{-} \bar{N}_{x}^{-}-\bar{\psi}_{x}^{-} \bar{N}_{y}^{-}-\frac{\alpha}{\operatorname{Re}} \bar{y} \bar{N}_{y}^{-}\right)+\frac{2 K}{\operatorname{Re}}\left(\bar{\psi}_{y y}^{-}+\bar{\psi}_{x x}^{--}\right)+\frac{(1+K / 2)}{\operatorname{Re}}\left(\bar{N}_{y y}^{--}+\bar{N}_{x x}^{--}\right)=0$

The boundary conditions can be written as:

$\bar{\psi}_{y}^{-}=0, \quad \bar{\psi}_{x}^{-}=-1, \quad \bar{N}=0, \quad$ at $\bar{y}=1$

$\bar{\psi}_{y}^{-}=0, \quad \bar{\psi}_{x}^{-}=1, \quad \bar{N}=0, \quad$ at $\bar{y}=-1$

\subsection{The similarity reductions of differential equation}

A group researchers derive the similarity solutions using the Lie group method [9]. The general solutions of this problem can be assumed as follows:

$\bar{\psi}=z(\bar{y}) Z(\bar{x}, \bar{y})$

$\bar{P}=P(\bar{x}, \bar{y})$

$\bar{N}=N(\bar{x}, \bar{y})$

Substituting (13) into (8), the equations become:

$-\frac{1+K}{\operatorname{Re}} \frac{d^{3} z}{d y^{3}}+\left(-z R_{1}-\frac{\alpha}{\operatorname{Re}} \bar{y}-3 \frac{1+K}{\operatorname{Re}} R_{2}\right) \frac{d^{2} z}{d y^{2}}+R_{1}\left(\frac{d z}{d y}\right)^{2}$

$+\left(z R_{3}+z R_{4}-\frac{\alpha}{\operatorname{Re}}-2 \frac{\alpha}{\operatorname{Re}} \bar{y} R_{2}-\frac{1+K}{\operatorname{Re}} R_{5}-3 \frac{1+K}{\operatorname{Re}} R_{6}\right) \frac{d z}{d \bar{y}}$

$-\left(\frac{\alpha}{\operatorname{Re}} R_{2}+\frac{\alpha}{\operatorname{Re}} \bar{y} R_{6}+\frac{1+K}{\operatorname{Re}} R_{9}-K R_{10}\right) z+\left(R_{7}-R_{8}\right) z^{2}+\frac{1}{H} \frac{\partial \bar{P}}{-}-\frac{1}{H} \frac{K}{\operatorname{Re}} \frac{\partial \bar{U}}{\partial \bar{y}}=0$
Where : $R_{1}=Z_{t}, \quad R_{2}=\frac{Z_{-}}{Z}, \quad R_{3}=Z_{---}, \quad R_{4}=\frac{Z_{-} Z_{-}}{Z}, \quad R_{5}=\frac{Z_{--}}{Z}$,

$R_{6}=\frac{Z_{--} y y}{Z}, \quad R_{7}=\frac{Z_{--} Z_{-}}{Z}, \quad R_{8}=\frac{Z_{--} Z_{-}}{Z}, \quad R_{9}=\frac{Z_{---}}{Z}$,

$R_{10}=\frac{Z_{--\bar{y}}}{Z}$.

The $z$ is a function of only $y$, and $Z 、 P$ and $U$ are functions of $x$ and $y$. From the formula (15) we come to the conclusion that must be a constant or a single variable function. Using the boundary conditions (11) - (12), we get:

$\bar{\psi}=\bar{x} G(\bar{y})$

$\bar{N}=\bar{x} M(\bar{y})$

Substituting (16) - (17) into (8) - (10), we obtain the following ordinary equations:

$(1+K) \frac{d^{4} G}{d y^{4}}+(\operatorname{Re} G+\alpha \bar{y}) \frac{d^{3} G}{d y^{3}}+2 \alpha \frac{d^{2} G}{d y^{2}}-\operatorname{Re} \frac{d G}{d \bar{y}} \frac{d^{2} G}{d y^{2}}+K \frac{d^{2} M}{d y^{2}}=0$
$\operatorname{Re} M \frac{d G}{d \bar{y}}-+(\operatorname{Re} G+\alpha \bar{y}) \frac{d M}{d \bar{y}}+2 K M+K \frac{d^{2} G}{d y^{2}}-\left(1+\frac{K}{2}\right) \frac{d^{2} G}{d y^{2}}=0$

The boundary conditions can be simplified as:

$\frac{d G(1)}{d \bar{y}}=0, \frac{d G(-1)}{d \bar{y}}=0, G(1)=1$

$M(1)=0, G(-1)=-1, M(-1)=0$

\section{VARIATION OF PARAMETERS METHOD}

To illustrate the basic concept of the variation of parameters method for differential equations, we consider the following general differential equation

$$
L u(x)+N u(x)+R u(x)=g(x)
$$

Where $L$ is a highest order linear operator, $R$ is a linear operator of order less then $L, N$ is a nonlinear operator, and $g(x)$ is a source term. By using the variation of parameters method as developed in some studies [10-12], we have the following general solution of Eq. (2) as

$u(x)=\sum_{i=0}^{n-1} \frac{A_{i} x^{i}}{i !}+\int_{0}^{x} \lambda(x, s)(-N u(s)-R u(s)+g(s)) d s$

Where $n$ is an order of given differential equation and $A_{i}$ are unknowns which can be further determined by initial/boundary conditions. Hence, we have the following for iterative scheme $\lambda(x, s)$ as

$$
\begin{array}{ll}
n=1, & \lambda(x, s)=1, \\
n=2, & \lambda(x, s)=x-s, \\
n=3, & \lambda(x, s)=\frac{x^{2}}{2 !}-x s+\frac{s^{2}}{2 !}, \\
n=4, & \lambda(x, s)=\frac{x^{3}}{3 !}-\frac{s x^{2}}{2 !}+\frac{s^{2} x}{2 !}-\frac{s^{3}}{3 !},
\end{array}
$$


Hence, we have the following iterative scheme (23), the equation (22) can be written as:

$u_{k+1}(x)=\sum_{i=0}^{n-1} \frac{A_{i} x^{i}}{i !}+\int_{0}^{x} \lambda(x, s)\left(-N u_{k}(s)-R u_{k}(s)+g(s)\right) d s, \quad k=0,1,2, \ldots$

What I want to emphasize is that the variation of parameters method (VPM) for solving system of four-order nonlinear system boundary value problems-similarity reductions of differential equation of micro-polar fluid.

\section{APPLICATION OF VPM}

Following the variation of parameters method and applying it on nonlinear system (18-19) we have following formula:

$G_{n+1}(y)=\left(A_{1}+A_{2} y+A_{3} \frac{y^{2}}{2}+A_{4} \frac{y^{3}}{6}\right)-$

$\int_{0}^{x}\left(\frac{x^{3}}{3 !}-\frac{s x^{2}}{2 !}+\frac{s^{2} x}{2 !}-\frac{s^{3}}{3 !}\right)\left(\frac{1}{K+1}\left(\left(\operatorname{Re} G_{n}+y\right) G_{n}^{\prime \prime \prime}(s)+2 \alpha G_{n}^{\prime \prime}(s)-\operatorname{Re} G_{n}^{\prime}(s) G_{n}^{*}(s)+K M_{n}^{\prime \prime}(s)\right)\right) d s$

$M_{n+1}(y)=\left(B_{1}+B_{2} y\right)-$

$\int_{0}^{x}(y-s)\left(\frac{1}{K / 2+1}\left(\left(\operatorname{Re} M_{n}(s)\right) G_{n}^{\prime}(s)-\left(\operatorname{Re} G_{n}(s)+\alpha y\right) M_{n}^{\prime}(s)+2 K M_{n}(s)+K G_{n}^{\prime \prime}(s)\right)\right) d s$

$G_{0}(y)=A_{2} y+A_{4} \frac{y^{3}}{6}$

$M_{0}(y)=B_{1}+B_{2} y$

Because the formula $M_{2}$ is too long, we don't write it out. Using the boundary conditions Eq. (20), we can get the value of A and B.

\section{CONCLUSIONS}

Using Variation of Parameters method (VPM), we obtain the approximate similarity solution of the nonlinear equations system of the flow of a micro-polar fluid through a porous channel with deforming walls. By our work, it is clear that VPM can successfully be applied to nonlinear equations like the one we have and the solutions are accurate, and they bear a remarkable resemblance. So that, it may conclude that VPM is an extremely efficient way to obtain the approximate analytical solutions for a system of non-linear differential equations.

\section{REFERENCES}

[1] Berman, S. 1953. Laminar flow in channels with porous walls. Journal of Applied Physics, 24, 1232-1235.

[2] Dauenhauer, C.E., Majdalani, J. 2003. Exact self-similarity solution of the Navier - Stokes equations for a porous channel with orthogonally moving walls. Physics of Fluids, 15 (6),1485-1495.

[3] White, F. 1991. Viscous Fluid Flow, McGraw-Hill, New York.

[4] Asghar, S., Mushtaq, M., Hayat, T. 2010. Flow in a slowly deforming channel with weak permeability: An analytical approach. Nonlinear Analysis Real World Applications, 11 (1), 555-561.

[5] Boutros, Y.Z., Abd-El-Malek, M.B., Tewfick, A.H. 1991. A formal expansion procedure for the internal solitary wave problem in a two-fluid system of constant topography. Acta Mechanica, 88 (3), 175-197.

[6] Abd-El-Malek, M.B., Badran, N.A., Hassan, H.S. 2002. Using group theoretic method to solve multi-dimensional diffusion equation. Journal of Computational and Applied Mathematics, 147 (2), 385-395.

[7] Si, X.H., Zheng, L.C., Zhang, X.X., Chao, Y. 2011. The flow of a micro-polar fluid through a porous channel with expanding or contracting walls. Central European Journal of Physics, 9 (3), 825-834.

[8] Srinivasacharya, D., Murthy, J.V.R., Venugopalam, D. 2001. Unsteady stokes flow of micro-polar fluid between two parallel porous plates. International Journal of Engineering Science, 39 (14), 1557-1563.

[9] Cao, L., Si, X., Zheng, L. 2015. The flow of a micro-polar fluid through a porous expanding channel: A Lie group analysis. Applied Mathematics and Computation, 270 (C), 242-250.

[10] Noor, M.A., Waheed, A., Al-Said, E. 2010. Modified variation of parameters method for solving system of second-order nonlinear boundary value problem. International Journal of Physical Sciences, 5 (16), 2426-2431.

[11] Noor, M.A., Moyud-Din, S.T., Waheed, A. 2008. Variation of Parameters Method for Solving Fifth-Order Boundary Value Problems. Applied Mathematics and Information Sciences, 2 (2), 135-141.

[12] Momani, S., Moadi, K., Noor, M.A. 2006. Decomposition method for solving a system of fourth-order obstacle boundary value problems. Applied Mathematics and Computation, 175 (2), 923-931. 